\title{
High prevalence of antinuclear antibodies in patients following venous thromboembolism
}

\author{
Joanna Natorska ${ }^{1, B-D}$, Magdalena Celińska-Löwenhoff ${ }^{2, D, E}$, Anetta I. Undas ${ }^{1, A, E, F}$ \\ ${ }^{1}$ Institute of Cardiology, Jagiellonian University Medical College, Kraków, Poland \\ ${ }^{2}$ Department of Internal Medicine, Jagiellonian University Medical College, Kraków, Poland \\ A - research concept and design; $B$ - collection and/or assembly of data; $C$ - data analysis and interpretation; \\ $D$ - writing the article; $E$ - critical revision of the article; $F$ - final approval of the article
}

Address for correspondence

Joanna Natorska

E-mail:j.natorska@szpitaljp2.krakow.pl

\section{Funding sources}

The study was supported by the gran

from the National Centre of Science

to UM0-2013/09/B/NZ5/00254 to AU.

\section{Conflict of interest}

None declared

Received on July 21, 2016

Reviewed on September 20, 2016

Accepted on 0ctober 6, 2017

\begin{abstract}
Background. Growing evidence suggests that activation of blood coagulation exerts protective functions during inflammation. However, it has been hypothesized that dysregulated immunothrombosis may lead to venous thromboembolism (VTE). Antinuclear antibodies (ANAs) are considered to promote the thrombotic tendency but there have been no reports on the association between ANAs and VTE.

Objectives. The objective of this study was to investigate if the presence of ANAs is associated with VTE.

Material and methods. We enrolled 283 consecutive patients, aged 18-66 years old, following a VTE episode, and 165 age-matched healthy controls. Patients with documented autoimmune diseases $(n=56$, 19.79\%), including antiphospholipid syndrome ( $n=48,16.9 \%)$, were excluded. Antinuclear antibodies were determined based on immunofluorescence. The specific immunofluorescence patterns observed at serum dilution equal to or greater than 1:100 were considered as positive ANAs.
\end{abstract}

Results. The final analysis included 227 patients (aged $41.07 \pm 11.4,98$ males, 129 females) following provoked $(n=111)$ or unprovoked $(n=116)$ VTE. Ninety-four $(42.2 \%)$ patients had positive ANAs, including 32 (33.3\%) with ANAs titer $\geq 1: 320$, whereas as few as 14 (8.4\%) controls had positive ANAs ( $p<0.007)$. ANA-positive patients were more commonly diagnosed with unprovoked $(n=55 ; 57.4 \%)$ than provoked VTE $(n=39 ; 40.6 \%)(p=0.03)$. A similar observation was made for ANAs titer $\geq 1: 320$ (26 patients with unprovoked vs 20 patients with provoked VTE, $p=0.04$ ). The presence of ANAs in the patient group did not correlate with age, sex, time since the VTE event, type of anticoagulation and its quality, inflammatory markers and D-dimer.

Conclusions. The prevalence of positive ANAs was 5 times higher among VTE patients than in controls. Antinuclear antibodies occur commonly in VTE and might be involved in the pathogenesis of unprovoked VTE.

Key words: venous thromboembolism, deep vein thrombosis, antinuclear antibodies

DOI

10.17219/acem/78361

\section{Copyright}

Copyright by Author(s)

This is an article distributed under the terms of the

Creative Commons Attribution Non-Commercial License

(http://creativecommons.org/licenses/by-nc-nd/4.0/) 


\section{Introduction}

Venous thromboembolism (VTE), encompassing deep vein thrombosis (DVT) and pulmonary embolism (PE), is a multifactorial disease. ${ }^{1}$ Venous thromboembolism is considered to be "provoked" in the presence of a temporary or reversible risk factor (such as surgery, trauma, immobilization, pregnancy, oral contraceptive use or hormone replacement therapy) within the last 6 weeks to 3 months before diagnosis, and "unprovoked" in the absence thereof. The identification of potential risk factors plays a crucial role in the decision-making process about the duration of therapy. Patients with antiphospholipid syndrome, those with a deficiency of antithrombin, protein $C$ or protein $S$, and patients homozygous for factor $\mathrm{V}$ Leiden or prothrombin G20210A (PTG20210A), may be candidates for indefinite anticoagulant treatment after a $1^{\text {st }}$ unprovoked VTE. ${ }^{2}$ Modern thrombophilia testing fails to identify any underlying prothrombotic tendency in a significant number of patients presenting with objectively confirmed VTE. ${ }^{3}$ Overall, $25-50 \%$ of patients with the first-time VTE have an idiopathic condition, without a readily identifiable risk factor. ${ }^{4}$

Correlative studies have hinted at a relationship between DVT and immune responses mainly associated with DNA and neutrophils leading to a concept of immunothrombosis. ${ }^{5}$ Growing evidence suggests that activation of blood coagulation exerts critical protective functions during inflammation. However, it has been hypothesized that dysregulated immunothrombosis may lead to VTE.

Antinuclear antibodies are considered to promote the thrombotic tendency directly by causing intimal damage due to their binding to the intimal cell wall and indirectly by causing a disorder of the intimal function due to their binding to intracellular structures of intimal cells such as the nucleus and cytoplasm. ${ }^{6,7}$ Unlike antiphospholipid antibodies, which bind to the cell wall in a restricted manner, ANAs bind to various cell components and may contribute to the thrombotic tendency by diverse mechanisms. Moreover, ANAs are involved in vascular disorders such as Raynaud's phenomenon. ${ }^{7}$

To the best of our knowledge, there have been no reports on the association between ANAs and VTE. Thus, the aim of our study was to investigate the prevalence of ANAs in patients following VTE.

\section{Material and methods}

\section{Patients}

We recruited 283 consecutive patients following documented provoked or unprovoked VTE (aged 18-66 years) who were referred to the Center for Coagulation Disorders in the John Paul II Hospital (Kraków, Poland) for further laboratory work-up between June 2013 and September 2015. Minimum time from the acute VTE episode prior to recruitment was 3 months. All the patients were treated with low molecular weight heparins followed by oral anticoagulants. The exclusion criteria were: acute coronary syndrome or stroke within the preceding 6 months, known malignancy, signs of acute infection or acute VTE, longterm antithrombotic treatment for other indications, e.g., atrial fibrillation or prosthetic cardiac valve implantation, pregnancy or postpartum period, end-stage kidney disease, documented autoimmune diseases ( $\mathrm{n}=56,19.79 \%$ ), including antiphospholipid syndrome (APS) diagnosed in 48 patients (16.9\%). Patients receiving anticoagulant therapy were eligible. The final analysis included 227 VTE patients. The Bioethics Committee of the Jagiellonian University approved the study. All the participants signed written informed consent.

The data on demographic characteristics, risk factors for VTE, comorbidities and medications was collected. The diagnosis of DVT of the lower or upper limb required a positive finding of color duplex sonography; an iliac/caval DVT was defined as abnormal duplex flow patterns typical of thrombosis or an intraluminal filling defect on contrast computed tomography or magnetic resonance venography. The diagnosis of PE was based on the presence of typical symptoms and positive results of high-resolution spiral computed tomography. Individuals with thrombosis at unusual sites were also diagnosed. Cerebral sinus venous thrombosis and hepatic/portal/splanchnic vein thrombosis was objectively documented using computed tomography angiography, magnetic resonance or magnetic resonance angiography.

Provoked VTE, i.e., associated with a known transient risk factor, was diagnosed in patients with VTE who experienced immobilization, surgery with the use of general anesthetic, major trauma, leg fracture or lower extremity plaster cast or hospitalization in the past month, intake of estrogen-containing oral contraceptives or hormone replacement therapy (HRT), as well as pregnancy or delivery within 3 months preceding the index event. Unprovoked (idiopathic) VTE episode was defined in patients who had no history of cancer, surgery, major trauma, plaster cast or hospitalization in the past month, pregnancy or delivery in the past 3 months.

A control population consisted of concurrent agematched healthy 165 participants.

\section{Laboratory tests}

After an overnight fast, venous blood was drawn from the antecubital vein into citrated tubes $(9: 1$ of $0.106 \mathrm{M}$ sodium citrate) and was centrifuged at $2500 \mathrm{~g}$ at $20^{\circ} \mathrm{C}$ for $10 \mathrm{~min}$ and stored in aliquots at $-80^{\circ} \mathrm{C}$ until analysis. Blood drawn into serum tubes was centrifuged at $2500 \mathrm{~g}$ at $-20^{\circ} \mathrm{C}$ for $10 \mathrm{~min}$ and stored at $-80^{\circ} \mathrm{C}$. To determine blood cell count, lipid profile, glucose, creatinine and thyroid-stimulating hormone (TSH), routine laboratory assays were used. High-sensitivity C-reactive protein (CRP) was determined using immunoturbidimetry (Roche Diagnostics, Mannheim, Germany). Fibrinogen was measured 
by the von Clauss method (Instrumentation Laboratory, Bedford, USA). Plasma D-dimer was measured with the Innovance D-dimer assay (Siemens, Marburg, Germany).

Antibodies directed at various cellular compartments including nuclear constituents, components of the nuclear envelope, mitotic spindle apparatus, cytosol, cytoplasmic organelles and cell membranes (ANAs) were tested by immunofluorescence (IIF) in IgG isotype (HEp2 cells and the conjugate; Euroimmun, Lübeck, Germany). Patients' sera were diluted with the cut-off 1:100 in PBS with Tween 20 (Sigma-Aldrich, St. Louis, USA), according to the international recommendations for the assessment of autoantibodies to cellular antigens. ${ }^{8}$ Visual determination of the staining pattern was assessed using the Nikon Eclipse E400 fluorescence microscope (Nikon Instruments Europe B.V., Amsterdam, Netherlands). The specific immunofluorescence patterns observed at serum dilution equal to or greater than 1:100 were considered as positive ANAs. An additional analysis for subjects with ANAs titer $\geq 1: 320$ was performed to confirm and strengthen the observations made for the whole ANA-positive patients' group.

The anti-DFS70 pattern was characterized by dense fine speckles distributed in the nucleus, confirmed in a reference laboratory of Euroimmun in Lübeck and subjected to semiquantitative enzyme-linked immunosorbent assay (ELISA) analysis (Euroimmun, Lübeck, Germany), according the manufacturer's instructions.

All patients were screened for thrombophilia, including the factor V Leiden mutation, prothrombin G20210A, as well as deficiency of antithrombin, protein $C$ or protein $\mathrm{S}$. Inherited thrombophilia was defined as the presence of either of the 2 mutations or a deficiency of 1 of the 3 coagulation inhibitors. Genotypes of FV Leiden (rs6025) and prothrombin G20210A (rs1799963) polymorphisms were ascertained by the allelic discrimination test using the TaqMan Genotyping assay on the ABI PRISM 7900HT Fast Real-Time PCR System (Life Technologies Co., Carlsbad, USA). Antithrombin anti-FX activity was measured using a chromogenic method in the presence of heparin (HemosIL ${ }^{\mathrm{TM}} \mathrm{TH}$, Instrumentation Laboratory SpA, Milan, Italy). Plasma PC was quantified using the HemosIL ${ }^{\mathrm{TM}}$ Protein $\mathrm{C}$ chromogenic assay (Instrumentation Laboratory $\mathrm{SpA}$, Milan, Italy). Free PS was quantified using the HemosIL ${ }^{\mathrm{TM}}$ Free Protein $S$ latex ligand immunoassay (Instrumentation Laboratory SpA, Milan, Italy).

To exclude APS based on the modified APS classification criteria by Miyakis et al., lupus anticoagulant (LA) was estimated using a clot-based assay, according to the recommendations published in 2009.9,10 Anticardiolipin and anti$\beta_{2}$ GP-I antibodies were determined by immunoenzymatic assays (INOVA Diagnostics, San Diego, USA). Reference ranges for IgG were up to 15 IgG phospholipid unit (GPL) and 8 standard IgG $\beta$-2 glycoprotein unit (SGU), respectively, and for IgM up to 17 IgM phospholipid unit (MPL) and 10 standard IgM $\beta-2$ glycoprotein unit (SMU), respectively. All positive cases were reevaluated after $12-16$ weeks. ${ }^{11}$
Serum protein profile was evaluated using serum protein electrophoresis (SPE) with the fully automated instrument Interlab G26 (Interlab, Rome, Italy) on agarose gel plates at $\mathrm{pH}$ 8.9. The final protein concentration ranged from $60 \mathrm{~g} / \mathrm{L}$ to $80 \mathrm{~g} / \mathrm{L}$. Densitometry of the pattern was determined for the relative quantification of protein zones.

\section{Statistical analysis}

Continuous variables were presented as median and interquartile range. Categorical variables were presented as the number and percentages. The Shapiro-Wilk test was used to test the normality of continuous variables. To examine the differences between the independent groups, the Kruskal-Wallis test was used. The $\chi^{2}$ or Pearson's exact tests were used for categorical variables. Statistical analysis was performed with STATISTICA v. 12.0 (StatSoft, Tulsa, USA).

\section{Results}

The demographic and laboratory data of the control and VTE patients are summarized in Table 1. Of the 165 control subjects, as few as 14 (8.4\%) individuals had positive ANAs, of whom $8(4.8 \%)$ had ANAs titer $=1: 100$ and $6(3.6 \%)$ had ANAs titer $=1: 320$.

Of the 227 VTE patients studied, 94 (42.2\%) individuals had positive ANAs, including 62 patients (64.6\%) with ANAs titer $<1: 320$ and 32 patients (33.3\%) with ANAs titer $\geq 1: 320$.

The prevalence of positive ANAs was 5 times higher among VTE patients compared with the controls (42.2\% vs $8.4 \%, \mathrm{p}=0.007)$. Of importance, patients with unprovoked VTE had positive ANAs more commonly $(\mathrm{n}=55 ; 57.4 \%)$ than those with provoked VTE $(n=39 ; 40.6 \%)(\mathrm{p}=0.03)$. A similar observation was made regarding the ANAs titer $\geq 1: 320$ (26 (27.1\%) patients with unprovoked vs 20 (20.8\%) patients with provoked VTE, $\mathrm{p}=0.04$ ).

The presence of ANAs was correlated with albumin $(\mathrm{r}=0.4, \mathrm{p}=0.014)$ and gamma globulin levels $(\mathrm{r}=0.5$, $\mathrm{p}=0.019$ ). The positive ANAs did not correlate with any other examined demographic or clinical variables like age, gender, body mass index (BMI), smoking, C-reactive protein (CRP), glucose, fibrinogen, D-dimer, time since last VTE event, as well as type of anticoagulation and its quality.

Anti-DFS70 antibodies were screened in 149 (90.3\%) control patients and $222(97.8 \%)$ VTE patients. There was no difference in the prevalence of anti-DFS70 antibodies in the control and VTE patients $(n=6(4.03 \%)$ vs $n=9$ $(4.05 \%)$, respectively, $\mathrm{p}=0.8)$. The DFS70 positive patients from the 2 groups did not differ in any of the examined demographic or clinical variables (data not shown).

The ANA-negative patients did not differ from ANA-positive patients in any of examined parameters such as lipid profile, glucose, CRP, fibrinogen, D-dimer, TSH or time since the VTE event. 


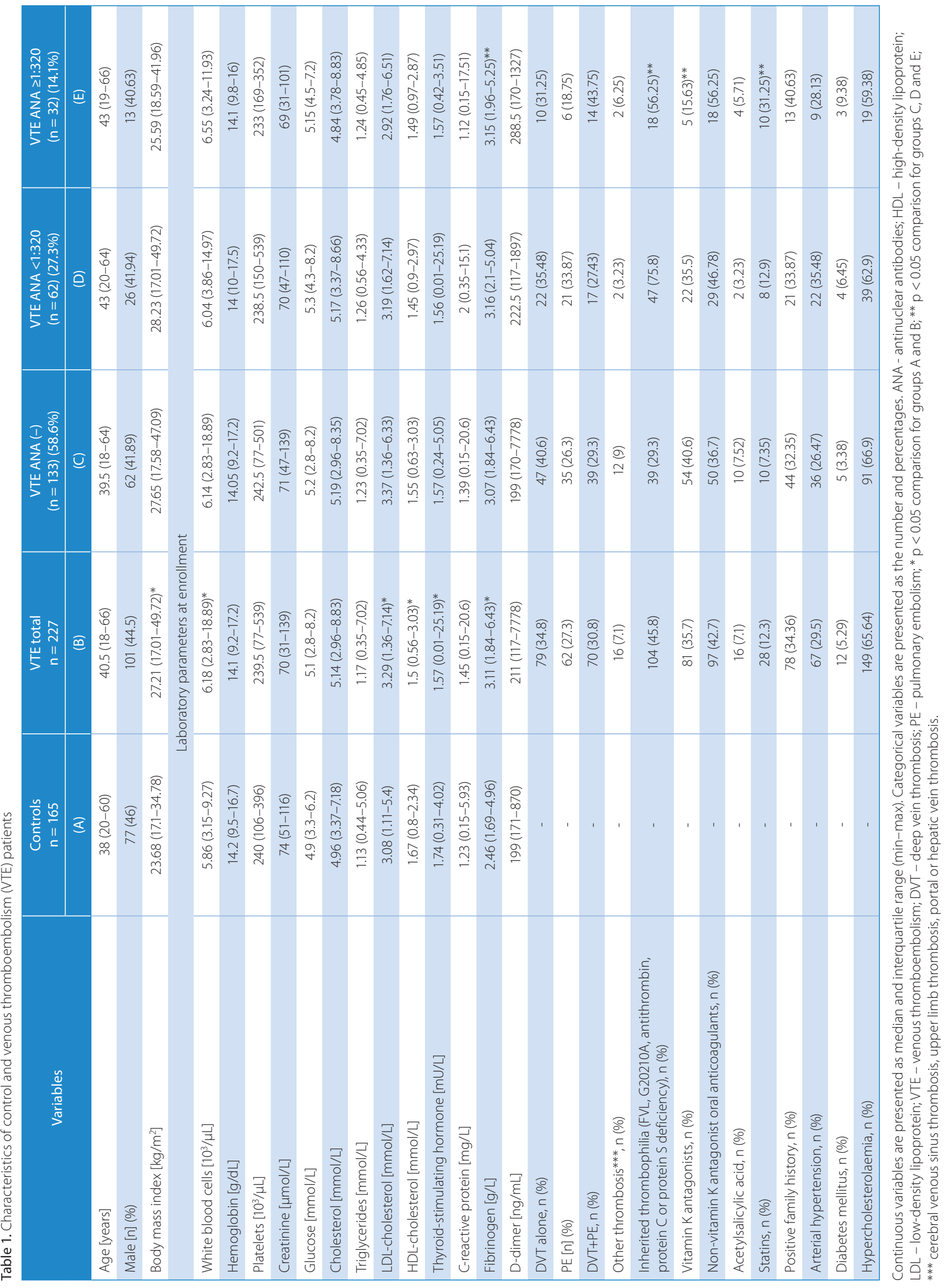




\section{Discussion}

To our knowledge, this study is the first to show that the presence of ANA among patients with VTE is higher than in control subjects. Based on this finding we hypothesize that ANA may play a potential role in the pathogenesis of VTE and might have a predictive value when it persists. Our rationale is that the individuals with autoimmune diseases are at a higher risk of VTE. However, this risk was mainly attributed to APS and/or exacerbation of autoimmune diseases, with the maximum rates within the first years since diagnosis, in particular in systemic lupus erythematosus (SLE) and anti-neutrophil cytoplasmic antibodies. ${ }^{12,13}$ Surprisingly, little is known about ANA in VTE patients without diagnosed autoimmune disease.

The link of APS to VTE is well established. ${ }^{14}$ Antiphospholipid syndrome is a complex autoimmune thrombotic disorder with defined clinical phenotypes, and experimental evidence suggests that antiphospholipid antibodies (aPLAs) play a causative role in vascular thrombosis and obstetric complications; however, the mechanisms are not well understood. ${ }^{15}$

Numerous mechanisms of thrombosis in the APS have been proposed. ${ }^{16}$ At least some of the variety of mechanisms may be explained by the spectrum of autoantibody. Mechanisms of autoantibody-mediated thrombosis may be divided into 2 general types: (1) autoantibodies interfere with hemostatic components occurring on membrane surfaces, for example the ability of certain autoantibodies to prolong in-vitro coagulation assays, and (2) autoantibody engagement of antigens on cell surfaces leads to transduction of a signal and altered cell activity. ${ }^{17,18}$

Moreover, several studies have reported that autoantibodies are associated with increased procoagulant activity on circulating blood monocytes. Tissue factor (TF), a major initiator of normal and pathological blood coagulation, can be expressed in larger amounts on monocytes and endothelial cells by a variety of stimuli, including circulating antibodies..$^{19}$ Moreover, monocyte-derived TF has been shown to be a key player in the initiation and propagation of VTE. ${ }^{5}$ It might be speculated that the presence of ANA may enhance TF expression and contribute to a low grade prothrombotic state, which increases the risk of thrombosis and/or its recurrence especially when additional thrombotic risk factors occur, e.g. immobilization, obesity or HRT.

A separate issue that needs to be addressed is the potential involvement of autoantibodies in immunothrombosis. It has been demonstrated that autoantibodies against beta2-glycoprotein I ( $\left.\beta_{2} \mathrm{GPI}\right)$ promote thrombin generation in vitro. ${ }^{19}$ This potentially sets up a vicious prothrombotic cycle and predisposes to VTE. It is, therefore, likely that other autoantibodies in a similar manner might lead to the activation of thrombotic mechanisms by endothelial cell damage and dysfunction. Since it is known that as few as $2-5 \%$ patients with elevated a PLAs levels do not develop thromboembolic events, it is possible that positive ANAs could also have some predictive value. ${ }^{20}$

ANA detected in our group of patients were unspecific; we did not find any disease-specific antibodies. The subset of autoantibodies most commonly found in "apparently healthy" individuals and several inflammatory nonrheumatic diseases are anti-DFS70 antibodies, directed against epithelium-derived growth factor $p 75$, which are usually absent in defined autoimmune diseases. ${ }^{21}$ Recently, positive anti-DFS70 antibodies have been shown to be associated with idiopathic VTE, arterial thrombosis and/or obstetric complications. ${ }^{22}$ In our study, however, we did not observe any association of anti-DFS70 antibodies and VTE, which might be explained by the analysis of patients below 65 years of age free of known autoimmune disorders. A potential role of anti-DFS70 antibodies in VTE requires further investigation.

Furthermore, it remains to be elucidated whether ANAs in VTE are primary or rather result from the disease itself, which is important for elucidating their role in thrombosis. Therefore, it might be useful to monitor the persistence of ANAs over a longer period of time.

It also is important to note that it is likely that by defining the potential relationship between ANAs and VTE, we might develop new diagnostic tools to identify patients at risk of VTE, especially unprovoked episodes.

In conclusion, our study indicates that positive ANAs, though at low titers, are common in young and middleaged patients following VTE, regardless of the time since the event, its type and anticoagulant therapy. More commonly, ANAs can be detected among patients after unprovoked VTE, which might suggest that autoimmune reactions could be involved in the pathogenesis of VTE. It would be also of interest to assess the role of ANAs as a potential risk factor of the VTE recurrence during a long-term follow-up in a large cohort study. Moreover, the predictive role of VTE in the development of symptomatic autoimmune disease in the future requires a further follow-up study.

\section{References}

1. Rosendaal FR. Venous thrombosis: A multicausal disease. Lancet. 1999;353:1167-1173.

2. Konstantinides SV, Torbicki A, Agnelli G, et al.; Task Force for the Diagnosis and Management of Acute Pulmonary Embolism of the European Society of Cardiology (ESC). 2014 ESC guidelines on the diagnosis and management of acute pulmonary embolism. Eur Heart $\mathrm{J}$. 2014;43:3033-3069.

3. Jenkins PV, Rawley O, Smith OP, O'Donnell JS. Elevated factor VIII levels and risk of venous thrombosis. Br J Haematol. 2012;157:653-663.

4. de Groot PG, Derksen B, Lisman T, Meijers JC, Rosendaal FR. Lupus anticoagulants and the risk of a first episode of deep venous thrombosis. J Thromb Haemost. 2005;3:1993-1997.

5. von Brühl ML, Stark K, Steinhart A, et al. Monocytes, neutrophils and platelets cooperate to initiate and propagate venous thrombosis in mice in vivo. J Exp Med. 2012;209:819-835.

6. Yamazaki M. Antiphospholipid antibody syndrome. In: Ichinose A, ed. Sciences of Thrombi, Hemostasis, and Angiology. Tokyo, Japan: Chugai-Igakusha; 2005:410-421. 
7. Kobayashi Y, Numano F. Angiitis. In: Morishita R, ed. Vascular Medicine. Tokyo, Japan: Medical Review; 2001:565-576.

8. Agmon-Levin N, Damoiseaux J, Kallenberg C, et al. International recommendations for the assessment of autoantibodies to cellular antigens referred to as anti-nuclear antibodies. Ann Rheum Dis. 2014;73:17-23.

9. Miyakis S, Lockshin MD, Atsumi T, et al. International consensus statement on an update of the classification criteria for definite antiphospholipid syndrome (APS). J Thromb Haemost. 2006;4:295-306.

10. Pengo V, Tripodi A, Reber G, et al.; Subcommittee on Lupus Anticoagulant/Antiphospholipid Antibody of the Scientific and Standardisation Committee of the International Society on Thrombosis and Haemostasis. Update of the guidelines for lupus anticoagulant detection. J Thromb Haemost. 2009;7:1737-1740.

11. Tripodi A, de Groot PE, Pengo V. Antiphospholipid syndrome: Laboratory detection, mechanisms of action and treatment. J Intern Med. 2011;270:110-122.

12. Aviña-Zubieta JA, Vostretsova K, De Vera MA, Sayre EC, Choi HK. The risk of pulmonary embolism and deep venous thrombosis in systemic lupuserythematosus: A general population-based study. Semin Arthritis Rheum. 2015;45:195-201.

13. Merkel PA, Lo GH, Holbrook JT, et al.; Wegener's Granulomatosis Etanercept Trial Research Group. High incidence of venous thrombotic events among patients with Wegener granulomatosis: The Wegener's Clinical Occurrence of Thrombosis (WeCLOT) study. Ann Intern Med. 2005;142:620-626.

14. Mustonen P, Lehtonen KV, Javela K, Puurunen M. Persistent antiphospholipid antibody (aPL) in asymptomatic carriers as a risk factor for future thrombotic events: A nationwide prospective study. Lupus. 2014;23:1468-1476.

15. Pierangeli SS, Chen PP, Raschi E, et al. Antiphospholipid antibodies and the antiphospholipid syndrome: Pathogenic mechanisms. Semin Thromb Hemost. 2008;34:236-250.

16. Roubey RA. Immunology of the antiphospholipid antibody syndrome. Arthritis Rheum. 1996;391:1444-1454.

17. Vlachoyiannopoulos PG, Routsias JG. A novel mechanism of thrombosis in antiphospholipid antibody syndrome. J Autoimmun. 2010;35:248-255.

18. Roubey RA, Hoffman M. From antiphospholipid syndrome to antibody-mediated thrombosis. Lancet. 1997;350:1491-1493.

19. Yalavarthi S, Gould TJ, Rao AN, et al. Release of neutrophil extracellular traps by neutrophils stimulated with antiphospholipid antibodies: A newly identified mechanism of thrombosis in the antiphospholipid syndrome. Arthritis Rheumatol. 2015;67:2990-3003.

20. Ortel TL. Thrombosis and the antiphospholipid syndrome. Hematology Am Soc Hematol Educ Program. 2005;1:462-468.

21. Basu A, Sanchez TW, Casiano CA. DFS70/LEDGFp75: An enigmatic autoantigen at the interface between autoimmunity, AIDS and cancer. Front Immunol. 2015;6:116. doi: 10.3389/fimmu.2015.00116

22. Marlet J, Ankri A, Charuel JL, et al. Thrombophilia associated with anti-DFS70 autoantibodies. PLoS One. 2015;10(9):e0138671. doi: 10.1371/journal.pone.0138671 